\title{
Infrared spectroscopy of jet-cooled neutral and ionized aniline-Ar
}

\author{
Hans Piest, ${ }^{\text {a) }}$ Gert von Helden, and Gerard Meijer \\ Department of Molecular and Laser Physics, University of Nijmegen, Toernooiveld, NL-6525 ED Nijmegen, \\ The Netherlands and FOM-Institute for Plasma Physics Rijnhuizen, Edisonbaan 14, \\ NL-3430 BE Nieuwegein, The Netherlands
}

(Received 23 September 1998; accepted 23 October 1998)

\begin{abstract}
We report the infrared (IR) absorption spectrum of the jet-cooled neutral aniline-Ar Van der Waals complex together with that of the aniline-Ar cation in the $350-1700 \mathrm{~cm}^{-1}$ range. The spectra are measured using mass-selective ion detection in two different IR-ultraviolet double-resonance excitation schemes, using a free-electron laser as a source of widely tunable, intense IR radiation. A comparison with calculated IR spectra of the bare neutral aniline and of the cation of aniline allows for an unambiguous assignment of all the observed modes. The dissociation limit of the neutral aniline-Ar complex is bracketed between 273 and $329 \mathrm{~cm}^{-1}$, significantly lower than previously estimated. (C) 1999 American Institute of Physics. [S0021-9606(99)02504-0]
\end{abstract}

\section{INTRODUCTION}

Over the last years a variety of experimental detection schemes has been demonstrated to determine vibrational spectra of gas-phase molecules with a sensitivity and a species selectivity that is substantially better than obtainable in conventional direct absorption spectroscopy. Infrared spectra of jet-cooled benzene have been obtained in an infraredultraviolet (IR-UV) double-resonance depletion scheme. ${ }^{1,2}$ Since then, this scheme has been exploited and developed further by others using either ion detection ${ }^{3-5}$ or fluorescence detection. ${ }^{6,7}$ Apart from using direct IR absorption, stimulated Raman pumping has been used in combination with UV-laser-based detection schemes to probe vibrational levels in jet-cooled molecules as well. ${ }^{8-10}$ Rather than measuring the IR transitions in depletion, selective background-free detection of IR-laser excited levels using nonresonant ionization has been demonstrated. ${ }^{11-13}$ In comparison to depletion spectroscopy the latter method has the advantage that it has an intrinsically better signal-to-noise ratio (SNR) in the spectrum, but it is less obvious how the observed IR line intensities in the spectrum reflect the IR absorption intensities as additional mode selectivity is present in the detection step. IR spectra have also been recorded via IR-laser-induced multiphoton dissociation (MPD), although the spectra thus obtained are often limited to the narrow (line-) tuning range of the $\mathrm{CO}$ and $\mathrm{CO}_{2}$ lasers employed for these studies. More versatile is a double-resonance MPD scheme in which, for instance, an intense $\mathrm{CO}_{2}$ laser or a UV laser induces selective dissociation of IR-laser excited vibrational levels, followed by chemiluminescence, laser-induced fluorescence (LIF) or resonance-enhanced multiphoton ionization (REMPI) detection of the dissociation products. ${ }^{14-17}$ For molecules in which ionization competes favorably with dissociation, like for the fullerenes and for various clusters of transition-metal atoms, IR-REMPI is a viable method to record IR spectra. ${ }^{18}$

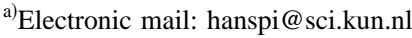

To extract accurate information on the vibrational frequencies of ionic species, the detection of threshold electrons, as in the zero kinetic energy (ZEKE) electron spectroscopy scheme, ${ }^{19,20}$ and the detection of the corresponding ions, as in the mass analyzed threshold ionization (MATI) scheme ${ }^{21}$ have proven to be extremely useful. In both the ZEKE and the MATI detection schemes transitions are made from vibrational levels in the neutral molecule to vibrational levels in the ion. To enable a direct comparison with theoretical calculations and to aid in the assignment of the vibrational modes, it is preferred to measure directly the IR absorption spectrum of the ion. A clever extension of the MATI scheme, in which an additional laser is used to induce a transition between Rydberg series converging to various bound levels in the ion ${ }^{22}$ approaches this goal. ${ }^{23}$ Alternatively, for weakly bonded ionic complexes such IR spectra can be measured using IR-laser-induced vibrational predissociation spectroscopy. ${ }^{24-26}$

In most studies reported to date in which tunable IR light is used, the region from about $3 \mu \mathrm{m}$ to shorter wavelengths is explored, thus covering the spectral region of the hydrogen-stretching motions and of the IR-active combination bands. To be able to explore the region of heavy-atom motion, a widely tunable light source at longer wavelengths is required. The unique performance characteristics of freeelectron lasers (FELs), in terms of light intensity and wavelength tunability, make them well suited for this purpose. In particular, when such a light source is combined with a "standard" molecular beam spectrometer, IR spectra of cold molecules can be measured with high sensitivity. In this paper we present the IR absorption spectrum of the jet-cooled aniline-Ar Van der Waals complex together with that of the aniline-Ar cation in the $350-1700 \mathrm{~cm}^{-1}$ region using a FEL as a source of IR radiation in two different IR-UV doubleresonance excitation schemes. Aniline-Ar is chosen as a model-system for these studies as both the bare aniline and the aniline-Ar Van der Waals complex have been extensively studied in the past and their spectroscopy is well known (see, for instance, Ref. 27 and Refs. 1-27 therein). 
From a comparison of the experimental spectra with calculated IR spectra, it is concluded that we indeed observe true IR spectra, both in line position and in line intensity, allowing for an unambiguous assignment of all the observed vibrational modes.

\section{EXPERIMENTAL SETUP}

Experiments are performed at the "Free-electron laser for infrared experiments" (FELIX) user facility in Nieuwegein, The Netherlands. ${ }^{28,29}$ FELIX produces pulsed IR radiation that is continuously tunable over the $40-2000 \mathrm{~cm}^{-1}$ range. The light output consists of macropulses of about $4 \mu \mathrm{s}$ duration containing up to $50 \mathrm{~mJ}$ of energy. Each macropulse consists of a train of micropulses that are $0.3-5$ ps long and $1 \mathrm{~ns}$ apart. In the present experiments, the macropulse repetition rate is $5 \mathrm{~Hz}$, and the bandwidth of the laser is typically set to $0.5 \%-1.0 \%$ of the central frequency.

The molecular beam spectrometer, including the two tunable pulsed UV laser systems used, is operated at a $10 \mathrm{~Hz}$ repetition rate and has been described previously. ${ }^{30}$ In short, a pulsed valve (R.M. Jordan Co.), operated with 2 bar Ar backing pressure, releases gas pulses of, typically, $30 \mu \mathrm{s}$ duration through a $0.5 \mathrm{~mm}$ diam orifice into vacuum of $10^{-6}$ Torr. A small amount of aniline is put in the sample compartment of the valve body, kept at a temperature of $40^{\circ} \mathrm{C}$, and is seeded in the carrier gas. About $4 \mathrm{~cm}$ downstream, the molecular beam is skimmed upon entering a differentially pumped Wiley-McLaren-type linear time-of-flight (TOF) mass spectrometer. The molecules in the beam interact with incoming UV laser beams as well as with the collimated FELIX beam (approximately 3-4 $\mathrm{mm}$ diam) at the crossing point of the mutually perpendicular molecular beam axis, laser beam axis, and TOF-tube axis. The maximum energy density of FELIX used in these experiments is about $0.2 \mathrm{~J} /$ $\mathrm{cm}^{2}$ per macropulse, which is reduced further by fixed-value attenuators if required. Ions produced in this region are either directly extracted and accelerated to the microchannel plate (MCP) detector using static electric fields, or they are first accumulated under field-free conditions and subsequently pulse extracted and accelerated to the MCP detector, yielding in either case mass spectra with a resolution of $M / \Delta M$ $\approx 200$. The signal from the MCP detector is amplified and fed into a $10 \mathrm{bit}, 100 \mathrm{Ms} / \mathrm{s}$ digital oscilloscope (LeCroy 9430) that is connected to a PC. The PC also controls the wavelength scanning of both UV lasers and of the freeelectron laser. A four-channel digital delay/pulse generator (SRS DG535) is used to synchronize the molecular beam spectrometer to the FELIX output to ns precision.

For the measurement of the IR spectrum of jet-cooled neutral aniline-Ar the IR-UV double-resonance depletion scheme, as schematically indicated in Fig. 1(A), is used. In this scheme the complexes are first irradiated with FELIX. If a vibrational transition is induced, vibrational predissociation can occur, provided the energy of the IR photon is higher than the binding energy $D_{0}$ of the complex. After the FELIX pulse is over, the IR-laser-induced depletion of aniline-Ar is monitored via one-color (1+1)-REMPI detection of aniline-Ar on the $S_{1} \leftarrow S_{0}$ origin band at $33976 \mathrm{~cm}^{-1} \cdot{ }^{27}$ As the molecular beam spectrometer runs at twice the repetition

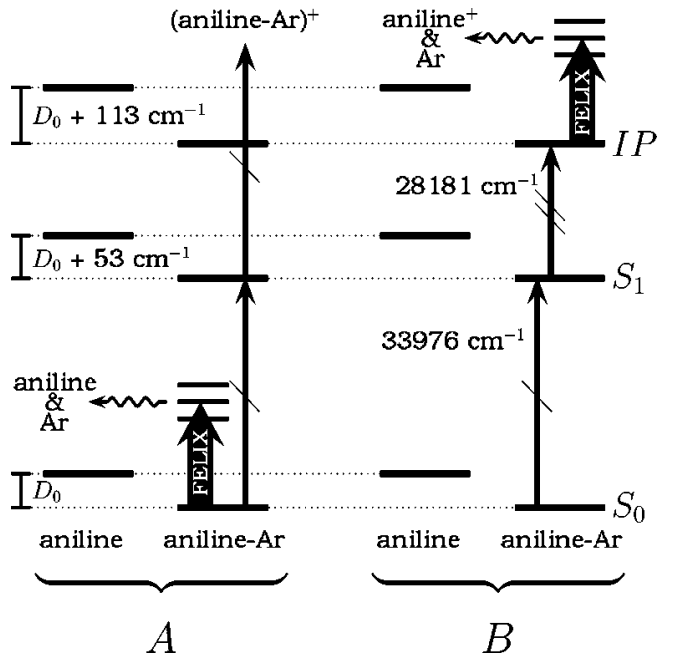

FIG. 1. Energy-level scheme for aniline and for aniline-Ar, indicating the two IR-UV double-resonance methods used. (A) Experimental scheme used to monitor the IR spectrum of neutral aniline-Ar. IR-laser-induced dissociation of ground-state aniline-Ar is detected via depletion of the UV-laserinduced ion signal at the mass of the complex. (B) Experimental scheme used to monitor the IR spectrum of the aniline-Ar cation. Aniline-Ar ions are produced in the vibrational ground-state via two-color $\left(1+1^{\prime}\right)$-REMPI of aniline-Ar in the absence of an electric field. IR-laser-induced dissociation of the ionic complex is detected via the appearance of the bare aniline cation, which is pulse extracted to the detector shortly after the end of the IR-laser pulse.

rate of FELIX, mass spectra both with and without the IRlaser present are measured by recording these during alternating shots.

For the measurement of the IR spectrum of the jetcooled aniline-Ar cation, the IR-UV double-resonance scheme as indicated in Fig. 1(B) is used. In this scheme, aniline-Ar ions are produced using two-color $\left(1+1^{\prime}\right)$-REMPI via the $S_{1} \leftarrow S_{0}$ origin band. The ions are produced in the absence of an electric field, and the energy of the ionizing photon is chosen such as to bring the Van der Waals complex just barely above the ionization threshold. With an excess energy of less than $10 \mathrm{~cm}^{-1}$, even the lowest Van der Waals mode of the complex ${ }^{31,32}$ cannot be excited. Therefore, ions will only be produced in the ground vibrational level, and will be about as cold as the neutral aniline-Ar complexes that they originate from. Shortly after production, the complex ions are irradiated with FELIX. If a vibrational transition is induced in the complex cation, vibrational predissociation can occur, leading to the appearance of aniline cations against zero background. After the FELIX pulse is over, all ions are pulse extracted towards the MCP detector, and mass selectively detected. When the ratio of the aniline cation signal intensity to the sum of the aniline-Ar and aniline cation intensity is monitored as a function of IR-laser frequency, i.e., when the fractional dissociation of the complex cation is monitored as a function of IR-laser frequency, a background-free measurement of the IR absorption spectrum of the internally cold aniline-Ar complex ion is performed. Recently, a similar scheme has been demonstrated for recording electronic spectra of cations of (poly)aromatic molecules. ${ }^{33}$ 


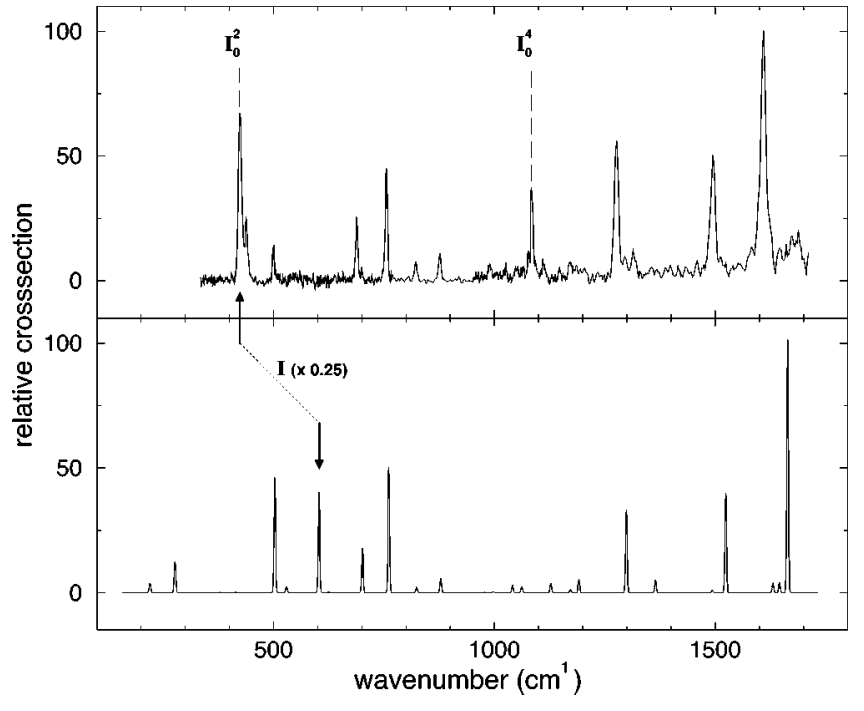

FIG. 2. Upper trace: Observed IR absorption spectrum of jet-cooled neutral aniline-Ar. The inversion mode $\left(I_{0}^{2}\right)$ and its overtone $\left(I_{0}^{4}\right)$ are assigned. Lower trace: Calculated IR absorption spectrum of neutral aniline. The inversion mode of aniline is not calculated correctly using the method employed. To make this mode less dominating in the spectrum it is scaled down in intensity by a factor 4 .

\section{RESULTS AND DISCUSSION}

In the upper part of Fig. 2 the measured IR absorption spectrum of jet-cooled aniline-Ar as recorded via the scheme depicted in Fig. 1(A) is shown. The spectrum is originally measured in depletion, but is transformed into an absorption spectrum to allow a direct comparison with the calculated IR absorption spectrum, shown in the lower part of Fig. 2. In the experiment, care is taken to avoid saturation of the IR depletion signal. In addition, it has been verified on the strongest transitions that the absorption intensity scales linearly with IR-laser intensity, and therefore, it is concluded that IR multiphoton excitation and/or sequential IR singlephoton absorption is not important under the present experimental conditions. The experimental spectrum is corrected for variations in the fluence of FELIX over the scan range. The spectrum is also corrected for slow fluctuations in the aniline-Ar content in the beam as well as for slow changes in the UV-laser-induced ionization efficiency during the recording of the spectrum by referencing the base line of the spectrum to the aniline-Ar ion signal intensity as measured in alternating shots, when FELIX is off. The observed widths of the lines in the spectrum are almost exclusively due to the linewidth of FELIX.

For comparison, we also performed IR-UV doubleresonance experiments on the bare aniline molecule, in that case detecting the depletion of the vibrational ground-state population of aniline via one-color $(1+1)$-REMPI at the electronic origin [around $34029 \mathrm{~cm}^{-1}$ (Ref. 34)] after IRlaser-induced population transfer to vibrationally excited levels. Similar spectra to the one shown in Fig. 2 were obtained in this way. A complication that now arises is that the UV laser will also be sensitive to vibrationally excited levels, when FELIX-induced "hot band transitions" coincide with the electronic origin, making the observed double-resonance signals harder to be interpreted unambiguously. In addition, the maximum attainable depth of the depletion signal in the bare aniline molecule can never reach the maximum attainable depth of $100 \%$ as in the complex, leading to an inferior SNR in the spectrum.

As a consequence, the method of depletion of the Van der Waals complex, often referred to as the "messenger method," appears to be the superior method to get information on the IR spectrum of the bare jet-cooled aniline molecule as well. Obviously, care has to be taken of the "matrix shift" due to the presence of the single Ar atom if one wants to extract accurate vibrational frequencies for the bare molecule from the spectra of the complex. Although these shifts are, in general, observed to be limited to a fraction of a percent of the corresponding frequency, ${ }^{27,31,32}$ significant effects have been reported for specific low-frequency out-ofplane vibrational modes in the $S_{1}$ state of aniline. ${ }^{35}$ Specific Van der Waals modes might combine with IR-active modes of the aniline molecule, leading to Van der Waals sidebands in the IR spectrum of the complex.

In the lower part of Fig. 2 the calculated IR absorption spectrum of neutral aniline is shown. The spectrum is calculated using the BECKE3LYP method ${ }^{36}$ with Dunning's D95(d,p) basis set $^{37}$ as implemented in GAUSSIAN $94 .{ }^{38}$ No scaling of the calculated frequencies is applied. With the standard $6-31 \mathrm{G}(2 \mathrm{~d}, \mathrm{p})$ basis set only slightly higher vibrational frequencies are obtained. The calculated stick spectrum is convoluted with a Gaussian line shape with a full width at half maximum (FWHM) of $5 \mathrm{~cm}^{-1}$. It has been well documented that aniline in the electronic ground state is distinctly nonplanar, with the plane of the phenyl group making an angle of approximately $38^{\circ}$ with the plane defined by the $\mathrm{NH}_{2}$ group, ${ }^{27}$ and that inversion of the $\mathrm{NH}_{2}$ group can be accurately described by a symmetric double-well potential, with a barrier to inversion of $526 \mathrm{~cm}^{-1} \cdot 39,40$ As frequencies and intensities are calculated in the harmonic approximation, the inversion mode of aniline as well as the possible occurrence of overtones of this inversion mode in the IR absorption spectrum will obviously not be reproduced correctly at all by the calculations. This is particularly misleading as this involves the strongest line in the calculated spectrum at 657 $\mathrm{cm}^{-1}$, which, for that reason, has been scaled down by a factor 4 in the spectrum displayed. This calculated frequency actually corresponds to the frequency in one half of the double-well potential, i.e., when an infinite barrier is assumed. Experimentally, it is known that the lowest energy levels for the inversion mode of aniline in the $S_{0}$ state are at 41, 424, and $700 \mathrm{~cm}^{-1} \cdot 39,40$ The strong IR absorption line that is seen at $423 \mathrm{~cm}^{-1}$ in the experimental absorption spectrum can, therefore, be safely assigned to the transition from the ground-state level to the lower component of the tunnelsplit $v=1$ level of the inversion motion, commonly referred to as the $I_{0}^{2}$ mode. On the high-frequency side of this mode a Van der Waals sideband is visible. With a frequency difference of $15 \mathrm{~cm}^{-1}$ this Van der Waals mode most likely corresponds to the symmetric (long-axis) bending mode of the complex. ${ }^{41}$ Apart from this $I_{0}^{2}$ mode, the only further discrepancy between the observed and calculated IR spectrum is a relatively strong peak in the experimental spectrum at 1084 $\mathrm{cm}^{-1}$, which is not present in the calculations. Using the 
TABLE I. Calculated vibrational frequencies (in $\mathrm{cm}^{-1}$ ) and integrated IR absorption intensities (in $10^{-18} \mathrm{~cm}^{2} \mathrm{~cm}^{-1}$ ) for neutral aniline and for the aniline cation. The symmetry label of the modes according to either $C_{s}$ symmetry (neutral) or $C_{2 v}$ symmetry (ion) is indicated. The observed frequencies (in $\mathrm{cm}^{-1}$ ) are deduced from the measurements of the IR spectra of the neutral and ionic aniline-Ar Van der Waals complex. The inversion mode of aniline, which is not calculated correctly, is indicated with ${ }^{1}$ in the table.

\begin{tabular}{|c|c|c|c|c|c|c|c|}
\hline \multicolumn{4}{|c|}{ Neutral } & \multicolumn{4}{|c|}{ Ion } \\
\hline \multirow[b]{2}{*}{$C_{s}$} & \multicolumn{2}{|c|}{ Calc. } & \multirow{2}{*}{$\begin{array}{l}\text { Obs. } \\
\nu_{\text {vib }}\end{array}$} & \multirow[b]{2}{*}{$C_{2 v}$} & \multicolumn{2}{|c|}{ Calc. } & \multirow{2}{*}{$\begin{array}{l}\text { Obs. } \\
\nu_{\text {vib }}\end{array}$} \\
\hline & $\nu_{\mathrm{vib}}$ & int. & & & $\nu_{\mathrm{vib}}$ & int. & \\
\hline$A^{\prime}$ & 220 & 1.1 & & $B_{1}$ & 184 & 1.4 & \\
\hline$A^{\prime \prime}$ & 277 & 3.4 & & $A_{2}$ & 365 & $\cdots$ & \\
\hline$A^{\prime \prime}$ & 379 & 0.0 & & $B_{2}$ & 387 & 0.5 & 386 \\
\hline$A^{\prime \prime}$ & 414 & 0.1 & & $B_{1}$ & 451 & 2.3 & 442 \\
\hline$A^{\prime}$ & 503 & 12.7 & 499 & $A_{1}$ & 524 & 0.2 & 519 \\
\hline$A^{\prime}$ & 529 & 0.7 & & $A_{2}$ & 558 & $\cdots$ & \\
\hline$A^{\prime}$ & $603^{1}$ & 44.6 & 424 & $B_{2}$ & 585 & 0.1 & \\
\hline$A^{\prime \prime}$ & 625 & 0.1 & & $B_{1}$ & $607^{1}$ & 29.3 & 652 \\
\hline$A^{\prime}$ & 701 & 4.9 & 688 & $B_{1}$ & 639 & 7.5 & 622 \\
\hline$A^{\prime}$ & 761 & 13.9 & 755 & $B_{1}$ & 794 & 9.2 & 785 \\
\hline$A^{\prime \prime}$ & 823 & 0.0 & & $A_{2}$ & 808 & $\ldots$ & \\
\hline$A^{\prime}$ & 824 & 0.6 & 822 & $A_{1}$ & 819 & 0.1 & \\
\hline$A^{\prime}$ & 879 & 1.6 & 867 & $B_{1}$ & 932 & 0.9 & 913 \\
\hline$A^{\prime \prime}$ & 960 & 0.0 & & $A_{1}$ & 982 & 0.0 & \\
\hline$A^{\prime}$ & 978 & 0.0 & & $A_{2}$ & 995 & $\cdots$ & \\
\hline$A^{\prime}$ & 998 & 0.1 & & $A_{1}$ & 1003 & 1.2 & 993 \\
\hline$A^{\prime}$ & 1041 & 0.9 & & $B_{1}$ & 1009 & 0.1 & \\
\hline$A^{\prime \prime}$ & 1062 & 0.7 & & $B_{2}$ & 1020 & 0.0 & \\
\hline$A^{\prime \prime}$ & 1128 & 1.0 & & $B_{2}$ & 1124 & 2.4 & 1107 \\
\hline$A^{\prime \prime}$ & 1172 & 0.3 & & $B_{2}$ & 1180 & 0.0 & \\
\hline$A^{\prime}$ & 1192 & 1.5 & & $A_{1}$ & 1201 & 0.0 & \\
\hline$A^{\prime}$ & 1299 & 9.1 & 1277 & $B_{2}$ & 1357 & 0.0 & \\
\hline$A^{\prime \prime}$ & 1354 & 0.0 & & $A_{1}$ & 1387 & 0.8 & \\
\hline$A^{\prime \prime}$ & 1365 & 1.4 & 1315 & $B_{2}$ & 1397 & 1.8 & 1360 \\
\hline$A^{\prime \prime}$ & 1494 & 0.3 & & $B_{2}$ & 1467 & 1.2 & 1434 \\
\hline$A^{\prime}$ & 1524 & 11.0 & 1496 & $A_{1}$ & 1509 & 11.9 & 1483 \\
\hline$A^{\prime \prime}$ & 1631 & 1.1 & & $B_{2}$ & 1544 & 2.1 & 1515 \\
\hline$A^{\prime}$ & 1646 & 1.1 & & $A_{1}$ & 1631 & 5.9 & 1583 \\
\hline$A^{\prime}$ & 1665 & 28.0 & 1610 & $A_{1}$ & 1671 & 19.2 & 1635 \\
\hline$A^{\prime}$ & 3172 & 2.5 & & $B_{2}$ & 3209 & 0.2 & \\
\hline$A^{\prime \prime}$ & 3173 & 0.4 & & $A_{1}$ & 3209 & 0.0 & \\
\hline$A^{\prime}$ & 3188 & 0.7 & & $A_{1}$ & 3220 & 0.0 & \\
\hline$A^{\prime \prime}$ & 3194 & 5.8 & & $B_{2}$ & 3230 & 0.4 & \\
\hline$A^{\prime}$ & 3212 & 2.1 & & $A_{1}$ & 3236 & 0.2 & \\
\hline$A^{\prime}$ & 3583 & 3.0 & & $A_{1}$ & 3557 & 46.9 & \\
\hline$A^{\prime \prime}$ & 3690 & 2.1 & & $B_{2}$ & 3681 & 16.3 & \\
\hline
\end{tabular}

parameters for the double-well potential that fit the three known experimental values for the energy levels of the inversion mode mentioned above, ${ }^{40}$ we calculated the next level to be at $1084.3 \mathrm{~cm}^{-1}$. Although this level has, to the best of our knowledge, never been observed, we feel confident in assigning the resonance at $1084 \mathrm{~cm}^{-1}$, correspondingly to the $I_{0}^{4}$ transition. All other modes in the experimental spectrum have a clear correspondence to modes in the calculated spectrum, both in frequency and in relative intensity, and one probably could even assign the relatively weak lines, in particular, in the $1000-1200 \mathrm{~cm}^{-1}$ region, to the corresponding features in the calculated spectrum. It appears that the calculated frequencies are on average approximately up to $3 \%$ too high. In Table I a complete list of all the calculated frequencies of the modes of aniline is given together with

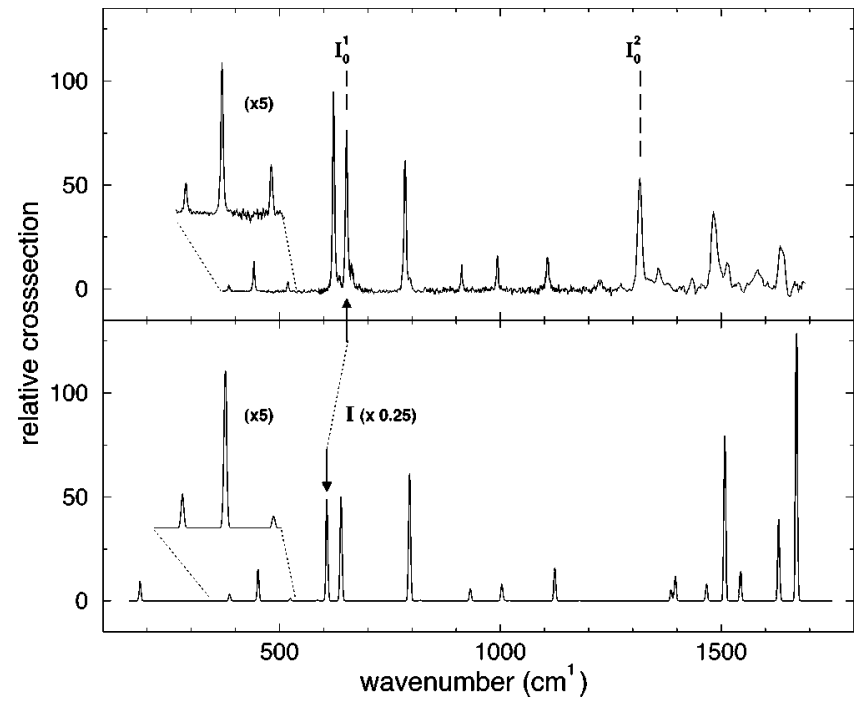

FIG. 3. Upper trace: Observed IR absorption spectrum of the aniline-Ar cation. The inversion mode $\left(I_{0}^{1}\right)$ and its overtone $\left(I_{0}^{2}\right)$ are assigned. Lower trace: Calculated IR absorption spectrum of the aniline cation. The geometry for the ion is planar ( $C_{2 v}$ symmetry). The inversion mode of aniline is not calculated correctly using the method employed. To make this mode less dominating in the spectrum it is scaled down in intensity by a factor 4 .

their symmetry label and their calculated IR absorption intensity. The assignment of the observed lines for aniline-Ar to the calculated modes is indicated in Table I. The accuracy of the experimentally determined frequencies is around $0.3 \%$ of these frequencies. We have chosen to omit the classification of the aniline modes according to the convention commonly used for benzene derivatives. ${ }^{42}$ From a visual inspection of the eigenmodes using a computer animation, it appears to us that for a substantial fraction of the modes such an assignment is not unambiguous, probably the reason for the widely varying assignments that have appeared throughout the literature. A more detailed study to uniquely establish such a classification, combining experimental IR absorption spectra with calculated spectra for various isotopomers of aniline, is underway.

In the upper part of Fig. 3 the observed IR absorption spectrum of the aniline-Ar cation as recorded via the scheme depicted in Fig. 1(B) is shown. Even though there are only on the order of 10-100 aniline-Ar ions produced per UVlaser pulse sequence, the background-free detection scheme that is used enables a spectrum to be recorded for the ion with a SNR that is substantially better than that for the corresponding neutral complex. The three strong lines in the $600-800 \mathrm{~cm}^{-1}$ region all have a weak Van der Waals sideband, approximately $12 \mathrm{~cm}^{-1}$ higher in energy, assigned to one of the bending modes of the complex. ${ }^{31,32}$ In no case a sideband at lower frequency is observed, thus explicitly demonstrating that the van der Waals cations are laser prepared in their vibrational ground state.

In the lower part of Fig. 3(B) the calculated IR absorption spectrum of the bare aniline ion is shown. The ion is known to be planar, and the calculation is performed using $C_{2 v}$ symmetry for the ion. The inversion vibration in the ion is still not accurately described by a harmonic potential, however, and the inversion mode will, therefore, not be ac- 
curately reproduced in the calculations. The inversion mode is calculated at $607 \mathrm{~cm}^{-1}$, and is again scaled down in intensity in the spectrum displayed. The inversion mode in the aniline cation is known to be at $656-658 \mathrm{~cm}^{-1}, 32,43$ and is found approximately $5 \mathrm{~cm}^{-1}$ lower in the aniline-Ar ion. ${ }^{31}$ We can thus identify the observed peak at $652 \mathrm{~cm}^{-1}$ with the $I_{0}^{1}$ transition in the complex ion. All the other observed peaks up to $1200 \mathrm{~cm}^{-1}$ can be readily identified with lines in the calculated spectrum. At higher frequencies the experimental spectrum suffers from a significantly lower IR laser intensity in combination with an increased spectral bandwidth of the IR-laser. It appears that the strongest lines in the calculated spectrum in this spectral region can still be identified in the experimental spectrum, although the spectrally integrated relative line intensities do not match as nicely as they do in the lower frequency part of the spectrum. The observed strong line at $1317 \mathrm{~cm}^{-1}$ is not reproduced in the calculations, and we assign this line to the $I_{0}^{2}$ transition; the overtone of the inversion mode in the aniline ion is known to be at $1323-1325 \mathrm{~cm}^{-1},{ }^{32,43}$ and is found approximately $10 \mathrm{~cm}^{-1}$ lower in the aniline-Ar ion. ${ }^{31}$ The two weak features at 1230 and $1270 \mathrm{~cm}^{-1}$, features that have no counterparts in the calculated spectrum either, might well be due to the overtone of the mode observed at $622 \mathrm{~cm}^{-1}$ and the combination of this mode with the inversion mode, respectively. A complete list of the calculated frequencies of the modes of the aniline cation is given in Table I, together with their symmetry assignment and their calculated integrated absorption cross section. The assignment of the observed lines for the complex cation is indicated in Table I.

It is seen in Fig. 3, and it is emphasized in the inset, that even at rather low frequencies clear resonances are observed, in particular, at 442 and at $386 \mathrm{~cm}^{-1}$. For both of these resonances, as well as for the strong resonance at $622 \mathrm{~cm}^{-1}$, the absolute value for the fraction of IR-laser-induced dissociation products is measured as a function of IR-laser intensity, the results of which are shown in Fig. 4. If absorption of a single IR photon leads to the appearance of aniline ion dissociation products, the aniline ion branching ratio will have a $[1-\exp (-\sigma I)]$ dependence on the IR-laser intensity $I$, with $\sigma$ the absorption cross section for the corresponding single-photon transition. In particular, for low values of the product $\sigma I$ the yield of aniline ions will depend linearly on the IR-laser intensity in this case. If sequential absorption of IR photons is required to overcome the dissociation limit, the dependence of the aniline ion yield on the laser intensity can be more complicated as it will, for instance, depend on the relaxation rate of the intermediate state, but it is, in any case, expected to show a higher-than-linear intensity dependence for low values of $\sigma I$. From the measured fractional dissociation curves shown in Fig. 4 it is concluded that the vibrational levels at 622 and at $442 \mathrm{~cm}^{-1}$ are located above the dissociation limit of the ionic aniline-Ar complex, whereas the level at $386 \mathrm{~cm}^{-1}$ is located below this limit in the ion.

This leads us to conclude that the dissociation limit $D_{0}$ of the neutral aniline-Ar Van der Waals complex is considerably lower than the previously reported values of around $400 \mathrm{~cm}^{-1}$, concluded upon from experiments bracketing the dissociation limit in the $S_{1}$ state of aniline between 442 and

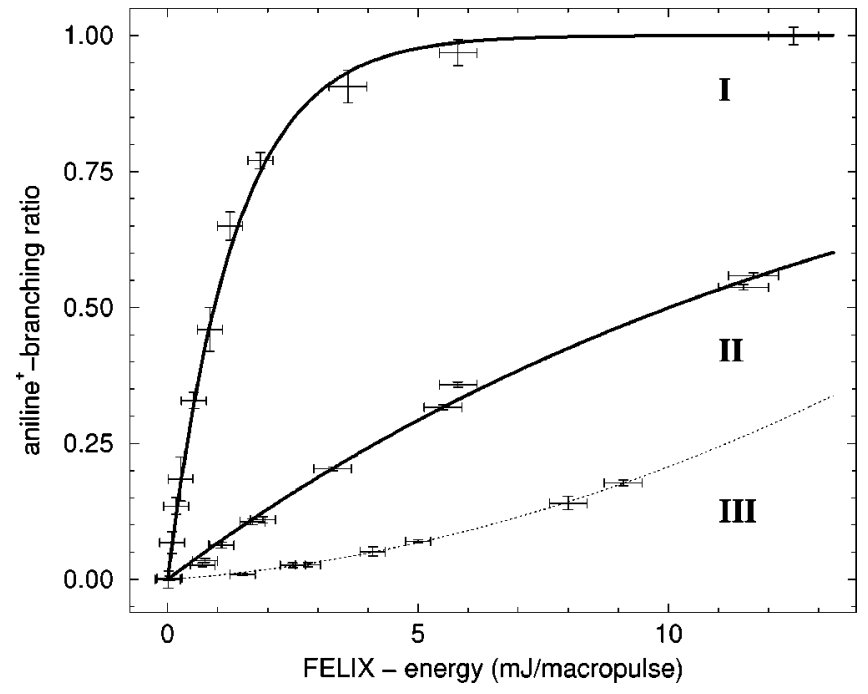

FIG. 4. Normalized yield of the IR-laser-induced aniline ion dissociation product as a function of IR-laser intensity for three different vibrational transitions in the aniline-Ar complex ion. The observed intensity dependence is fitted to a $[1-\exp (-\sigma I)]$ dependence for excitation on the modes at 622 and $442 \mathrm{~cm}^{-1}$, curves I and II, respectively. A higher than linear intensity dependence of the aniline ion yield is observed for excitation on the mode at $386 \mathrm{~cm}^{-1}$, curve III.

$460 \mathrm{~cm}^{-1} \cdot{ }^{44}$ Using a measured value of $-(113 \pm 2) \mathrm{cm}^{-1}$ for the shift of the ionization potential of aniline upon complexation with $\mathrm{Ar}$, in good agreement with values reported by others, ${ }^{31,32,45}$ the bracketing of the dissociation limit in the Van der Waals cation between 386 and $442 \mathrm{~cm}^{-1}$ implies a dissociation limit $D_{0}$ for the neutral aniline-Ar Van der Waals complex above $273 \mathrm{~cm}^{-1}$ and below $329 \mathrm{~cm}^{-1}$. Although this upper limit is rather low relative to other reports as well as to model calculations, ${ }^{41}$ it is important to note that all previous experimental upper and lower limits for $D_{0}$ have been obtained via monitoring the vibrational predissociation in the $S_{1}$ state of the complex. When the time scale for vibrational predissociation in this electronically excited state is substantially longer than the radiative lifetime of this state [around 7 ns (Ref. 27)], vibrational predissociation will not be noticed. In both experimental schemes that we have employed, on the other hand, the vibrational predissociation is allowed to occur on a time scale that is three orders of magnitude longer ( $\mu$ s time scale), and it is, therefore, not unexpected that a more stringent upper limit for the dissociation limit is found. It is interesting to note that a similar situation exists for the benzene-Ar complex, where sophisticated experimental measurements point to an upper limit for $D_{0}$ of $340 \mathrm{~cm}^{-1}$, significantly lower than theoretical results, and where it is remarked that "as a general trend all theoretical results are larger than the experimental upper limits.",46

From the measured curves as displayed in Fig. 4, it is in principle possible to determine absolute IR absorption cross sections. For this, the spectral profile of the laser, the spatial profile of the IR beam, and its overlap with the ionic Van der Waals complexes produced by the UV lasers need to be accurately known, in addition to the laser pulse energy. This is further complicated by the motion of the molecules into and out of the laser interaction region on the time scale of the experiment. For now, we can only conclude that the absolute values for the cross section resulting from the measurements 
agree with the calculated values within an order of magnitude. Experiments aimed at determining the absolute absorption cross sections more precisely are currently underway.

\section{CONCLUSIONS}

Combining a "standard" molecular beam apparatus with the unique performance characteristics of an intense and widely tunable IR free-electron laser yields a versatile spectrometer, capable of measuring IR spectra of internally cold aromatic molecules and their complexes. In the two IR-UV double-resonance schemes employed in this study, true IR absorption spectra of jet-cooled aniline-Ar complexes are obtained, greatly facilitating the spectral assignment as both line positions and line intensities can be compared to calculated spectra. It is observed that the calculated frequencies are on average slightly too high, up to $3 \%$, and that the relative line intensities are well reproduced. Both in the IR spectrum of neutral and cationic aniline-Ar the $\mathrm{NH}_{2}$ inversion mode and its overtones, which cannot be reproduced correctly at all in the calculations, are observed to be important. It is concluded that the dissociation limit $D_{0}$ of the aniline-Ar Van der Waals complex is between 273 and 329 $\mathrm{cm}^{-1}$, considerably lower than previously estimated. The double-resonance techniques demonstrated here on the aniline-Ar model system, hold great promise for the study of optical properties of jet-cooled (poly-) aromatic hydrocarbons (PAHs), in general. Among other things, such studies might yield the required laboratory data to be able to draw firm conclusions on the possible role of these PAHs in astrophysics.

\section{ACKNOWLEDGMENTS}

The authors gratefully acknowledge the support by the "Stichting voor Fundamenteel Onderzoek der Materie (FOM)'" in providing the required beam time on FELIX and highly appreciate the skillful assistance by the FELIX staff, in particular, Dr. A. F. G. van der Meer, and the expert technical assistance by A. J. A. van Roij. This research is part of the research program of FOM and is in part financially supported by the Council for Chemical Sciences, both of which are financially supported by the "Nederlandse Organisatie voor Wetenschappelijk Onderzoek (NWO)," and receives direct support by the NWO via PIONIER Grant No. 030-66-89.

${ }^{1}$ R. H. Page, Y. R. Shen, and Y. T. Lee, J. Chem. Phys. 88, 5362 (1988).

${ }^{2}$ R. H. Page, Y. R. Shen, and Y. T. Lee, J. Chem. Phys. 88, 4621 (1988).

${ }^{3}$ S. Tanabe, T. Ebata, M. Fujii, and N. Mikami, Chem. Phys. Lett. 215, 347 (1993).

${ }^{4}$ R. N. Pribble and T. S. Zwier, Science 265, 75 (1994).

${ }^{5}$ R. N. Pribble, F. C. Hagemeister, and T. S. Zwier, J. Chem. Phys. 106, 2145 (1997).

${ }^{6}$ Th. Walter, H. Bitto, T. K. Minton, and J. R. Huber, Chem. Phys. Lett. 231, 64 (1994).

${ }^{7}$ T. Ebata, N. Mizuochi, T. Watanabe, and N. Mikami, J. Phys. Chem. 100, 546 (1996).

${ }^{8}$ P. Esherick, A. Owyoung, and J. Plivia, J. Chem. Phys. 83, 3311 (1985).
${ }^{9}$ G. V. Hartland, B. F. Hanson, V. A. Venturo, and P. M. Felker, J. Phys. Chem. 96, 1164 (1992).

${ }^{10}$ T. Ebata, T. Watanabe, and N. Mikami, J. Phys. Chem. 99, 5761 (1995).

${ }^{11}$ T. Omi, H. Shitomi, N. Sekiya, K. Takazawa, and M. Fujii, Chem. Phys. Lett. 252, 287 (1996).

${ }^{12}$ M. Putter, G. von Helden, and G. Meijer, Chem. Phys. Lett. 258, 118 (1996).

${ }^{13}$ S. Ishiuchi, H. Shitomi, K. Takazawa, and M. Fujii, Chem. Phys. Lett. 283, 243 (1998)

${ }^{14}$ L. I. Yeh, M. Okumura, J. D. Myers, J. M. Price, and Y. T. Lee, J. Chem. Phys. 91, 7319 (1989).

${ }^{15}$ R. D. F. Settle and T. R. Rizzo, J. Chem. Phys. 97, 2823 (1992).

${ }^{16}$ O. V. Boyarkin and T. R. Rizzo, J. Chem. Phys. 103, 1985 (1995).

${ }^{17}$ M. Hippler and M. Quack, J. Chem. Phys. 104, 7426 (1996).

${ }^{18}$ G. von Helden, I. Holleman, G. M. H. Knippels, A. F. G. van der Meer, and G. Meijer, Phys. Rev. Lett. 79, 5234 (1997).

${ }^{19}$ K. Müller-Dethlefs, M. Sander, and E. W. Schlag, Z. Naturforsch. A 39, 1089 (1984).

${ }^{20}$ K. Müller-Dethlefs and E. W. Schlag, Annu. Rev. Phys. Chem. 42, 109 (1991).

${ }^{21}$ L. Zhu and P. M. Johnson, J. Chem. Phys. 94, 5769 (1991).

${ }^{22}$ D. P. Taylor, J. G. Goode, J. E. LeClaire, and P. M. Johnson, J. Chem. Phys. 103, 6293 (1995).

${ }^{23}$ A. Fujii, A. Iwasaki, T. Ebata, and N. Mikami, J. Phys. Chem. A 101, 5963 (1997).

${ }^{24}$ M. Okumura, L. I. Yeh, and Y. T. Lee, J. Chem. Phys. 83, 3705 (1985).

${ }^{25}$ M. Okumura, L. I. Yeh, and Y. T. Lee, J. Phys. Chem. 94, 3416 (1990).

${ }^{26}$ R. V. Olkhov, S. A. Nizkorodov, and O. Dopfer, J. Chem. Phys. 108, 10046 (1998).

${ }^{27}$ W. E. Sinclair and D. W. Pratt, J. Chem. Phys. 105, 7942 (1996).

${ }^{28}$ D. Oepts, A. F. G. van der Meer, and P. W. van Amersfoort, Infrared Phys. Technol. 36, 297 (1995).

${ }^{29}$ G. M. H. Knippels, R. F. X. A. M. Mols, A. F. G. van der Meer, D. Oepts, and P. W. van Amersfoort, Phys. Rev. Lett. 75, 1755 (1995).

${ }^{30}$ M. G. H. Boogaarts, G. von Helden, and G. Meijer, J. Chem. Phys. 105, 8556 (1996).

${ }^{31}$ M. Takahashi, H. Ozeki, and K. Kimura, J. Chem. Phys. 96, 6399 (1992).

${ }^{32}$ X. Zhang, J. M. Smith, and J. L. Knee, J. Chem. Phys. 97, 2843 (1992).

${ }^{33} \mathrm{Ph}$. Brechignac, Faraday Discussions, Number 109, Chemistry and Physics of Molecules and Grains in Space, edited by The Faraday Division (Royal Society of Chemistry, London, 1998).

${ }^{34}$ E. R. Th. Kerstel, M. Becucci, G. Pietrapeizia, and F. Castellucci, Chem. Phys. 199, 263 (1995).

${ }^{35}$ B. Coutant and Ph. Brechignac, J. Chem. Phys. 100, 7087 (1994).

${ }^{36}$ A. D. Becke, J. Chem. Phys. 98, 5648 (1993).

${ }^{37}$ T. H. Dunning, Jr. and P. J. Hay, in Modern Theoretical Chemistry, edited by H. F. Schäfer III, (Plenum, New York, 1976).

${ }^{38}$ Gaussian 94, Revision D.3, M. J. Frisch, G. W. Trucks, H. B. Schlegel, P. M. W. Gill, B. G. Johnson, M. A. Robb, J. R. Cheeseman, T. Keith, G. A. Petersson, J. A. Montgomery, K. Raghavachari, M. A. Al-Laham, V. G. Zakrzewski, J. V. Ortiz, J. B. Foresman, J. Cioslowski, B. B. Stefanov, A. Nanayakkara, M. Challacombe, C. Y. Peng, P. Y. Ayala, W. Chen, M. W. Wong, J. L. Andres, E. S. Replogle, R. Gomperts, R. L. Martin, D. J. Fox, J. S. Binkley, D. J. Defrees, J. Baker, J. P. Stewart, M. Head-Gordon, C. Gonzales, and J. A. Pople, Gaussian, Inc., Pittsburgh, PA, 1995.

${ }^{39}$ M. Quack and M. Stockburger, J. Mol. Spectrosc. 43, 87 (1972).

${ }^{40}$ R. A. Kydd and P. J. Krüger, Chem. Phys. Lett. 49, 539 (1977).

${ }^{41}$ P. Parneix, N. Halberstadt, Ph. Brechignac, F. G. Amar, A. van der Avoird, and J. W. I. van Bladel, J. Chem. Phys. 98, 2709 (1993).

${ }^{42}$ G. Varsanyi, Assignments for Vibrational Spectra of Seven Hundred Benzene Derivations (Wiley, New York, 1974).

${ }^{43}$ X. Song, M. Yang, E. R. Davidson, and J. P. Reilly, J. Chem. Phys. 99, 3224 (1993)

${ }^{44}$ M. R. Nimlos, M. A. Young, E. R. Bernstein, and D. F. Kelley, J. Chem. Phys. 91, 5268 (1989).

${ }^{45}$ S. Douin, P. Hermine, P. Parneix, and Ph. Brechignac, J. Chem. Phys. 97, 2160 (1992).

${ }^{46}$ H. Krausse and H. J. Neusser, J. Chem. Phys. 99, 6278 (1993). 